\title{
Perinephric abscess secondary to a staghorn calculus presenting as a subcutaneous abscess
}

\author{
Dylan Tsukagoshi, MB BS; Bozanka Dinkovski, MB BS; Sunil Dasan, MB BS, FFAEM; \\ Jalpesh Jethwa, MB BS
}

\begin{abstract}
Perinephric abscesses often present with flank pain and signs of sepsis, and are known to discharge to the skin. We report a case of a young patient presenting with a 48-hour history of painful swelling in her right renal angle following a urinary tract infection and no overt signs or symptoms of sepsis. Plain abdominal films showed a perinephric abscess secondary to a staghorn calculus in the upper pole of the right kidney. On incision and drainage, $700 \mathrm{~mL}$ of pus was drained freely. The patient recovered well but required partial nephrectomy.

Subcutaneous abscess as a result of intra-abdominal pathology is a recognized but rare complication. This case illustrates that subcutaneous abscesses may have underlying intra-abdominal pathology without overt signs of sepsis.
\end{abstract}

Key words: perinephric; subcutaneous; abscess; calculus

\section{RÉSUMÉ}

Les phlegmons périnéphrétiques s'accompagnent souvent de douleur au flanc et de signes de septicémie et sont connus pour suppurer vers la peau. Nous présentons le cas d'une jeune patiente souffrant de symptômes d'œdème douloureux au rein droit depuis 48 heures à la suite d'une infection urinaire non accompagnés de signes ni de symptômes apparents de septicémie. Les clichés abdominaux simples révélèrent un phlegmon périnéphrétique attribuable à un calcul coralliforme dans le pôle supérieur du rein droit. Lors de l'incision et du drainage, $700 \mathrm{~mL}$ de pus s'écoulèrent librement. La patiente se rétablit complètement, mais elle dut subir une néphrectomie partielle.

L'abcès sous-cutané résultant d'une pathologie intra-abdominale est une complication reconnue mais rare. Le présent cas démontre que les abcès sous-cutanés peuvent cacher une pathologie intra-abdominale sous-jacente sans aucun signe apparent de septicémie.

\section{Case report}

A 21-year-old woman presented to the emergency department with a 48-hour history of an increasingly painful $4 \times 4-\mathrm{cm}$ fluctuant swelling in her right lumbar region, with bruising. Two weeks previously, she had been treated for a urinary tract infection, which had resolved com- pletely with antibiotic management. There was no history of diabetes, injection drug use or lumbar trauma.

On examination she appeared well, with a temperature of $37.0^{\circ} \mathrm{C}$, blood pressure of $120 / 50 \mathrm{~mm} \mathrm{Hg}$, arterial oxygen saturation of $98 \%$ on air, and blood glucose 6.7 $\mathrm{mmol} / \mathrm{L}$. She was tachycardic with a pulse of 130 beats/min, but her abdomen was soft and non-tender. The 
swelling in the right renal angle was soft, fluctuant and well demarcated, with mottling. Results of bedside urinalysis showed leucocytes and a trace of blood.

A diagnosis of subcutaneous abscess was made, and incision and drainage was performed under local anesthetic, during which $700 \mathrm{~mL}$ of offensive-smelling pus drained freely. Swabs and cultures were sent, including acid-fast bacilli, and the wound was left to drain into a stoma bag. Over the next 3 days, $800 \mathrm{~mL}$ of additional pus mixed with urine was drained.

An abdominal x-ray (Fig. 1) showed a right renal staghorn calculus, absent right renal outline and increased shadowing in the renal space. Abdominal CT (Fig. 2) confirmed a staghorn calculus in the right renal pelvis with an associated perinephric abscess extending into the right posterolateral abdominal wall and extending inferiorly to the iliac crest. A massive liver without any obvious focal lesion was also seen. Ultrasound showed the abscess was loculated. Laboratory investigations showed a hemoglobin $6.4 \mathrm{~g} / \mathrm{dL}$, white cell count 12.2 with neutrophilia and platelet count 946000 cells $/ \mathrm{mm}^{3}$. Proteus spp. were isolated from the drained pus. A blood transfusion and intravenous antibiotic therapy were commenced but, because of the complexity of the abscess, percutaneous drainage was withheld until drainage through the wound had ceased.

The abnormal investigations and hepatomegaly im-

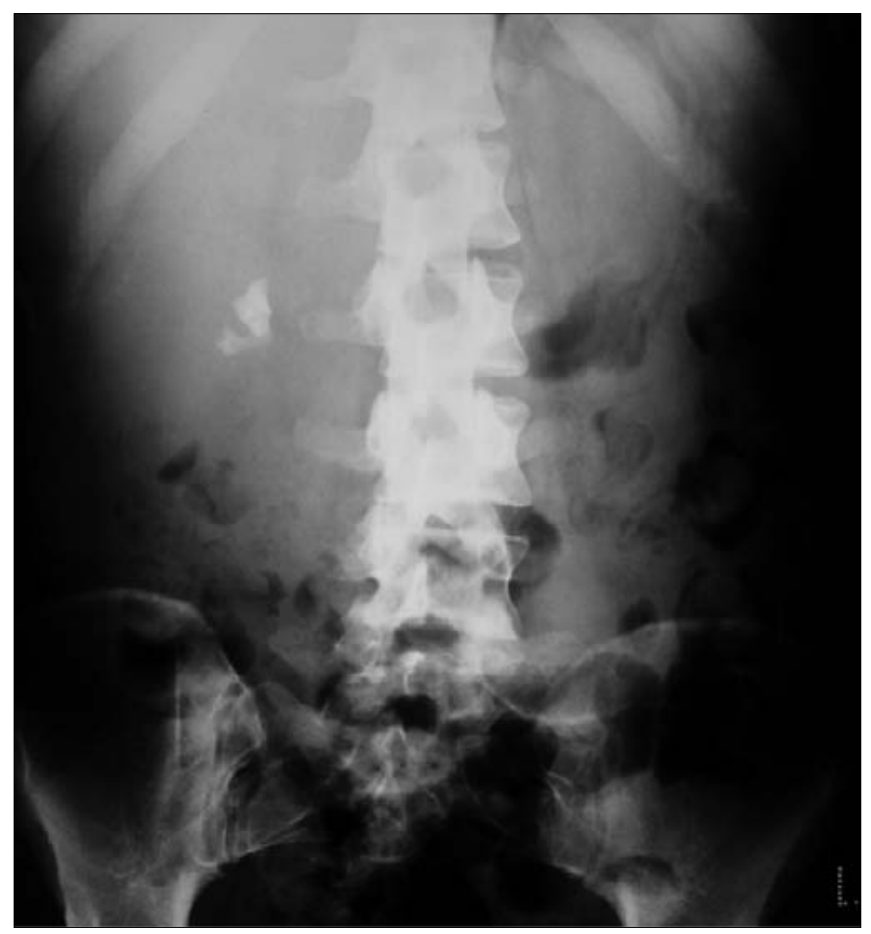

Fig. 1. Results of $x$-ray of the right kidney and ureter, and the bladder, showing increased opacity in the right renal angle and a staghorn calculus. proved following percutaneous drainage of the abscess, and were believed to be the result of chronic infection. A follow-up CT scan performed 4 weeks after initial presentation showed a small residual collection of pus and the previously identified staghorn calculus. Contrast CT showed absent perfusion to the upper pole of the kidney, and the patient was referred for a partial nephrectomy.

\section{Discussion}

Perinephric abscess is defined as a collection of suppurative material in the perinephric space between Gerota's fascia and the renal capsule. Over $80 \%$ occur secondary to renal tract calculi with ascending urinary tract infections, most commonly due to Escherichia coli and Proteus species. More rarely, abscesses develop via hematogenous spread; in these cases, Staphylococcus aureus is the most common causative organism. Other important organisms known to cause perinepheric abscesses include gram-negative bacteria such as Klebsiella, Enterobacter, Pseudomonas and Serratia; anaerobes such as Clostridium, Bacteroides and Actinomyces and fungi such as Candida. Mycobacterium tuberculosis as a cause is now rare. There is no correlation between the presence of kidney stones and the microbe isolated. ${ }^{1}$

Diabetes mellitus and previous urological surgery are known pre-disposing factors. ${ }^{2}$ Other risk factors include a history of pyelonephritis, urinary calculi, renal or ureteric obstruction, and neurologic impairment (e.g., neurogenic bladder). ${ }^{3}$ Perinephric abscesses are more common in children, the immunologically compromised, and after lithotripsy or renal transplant.

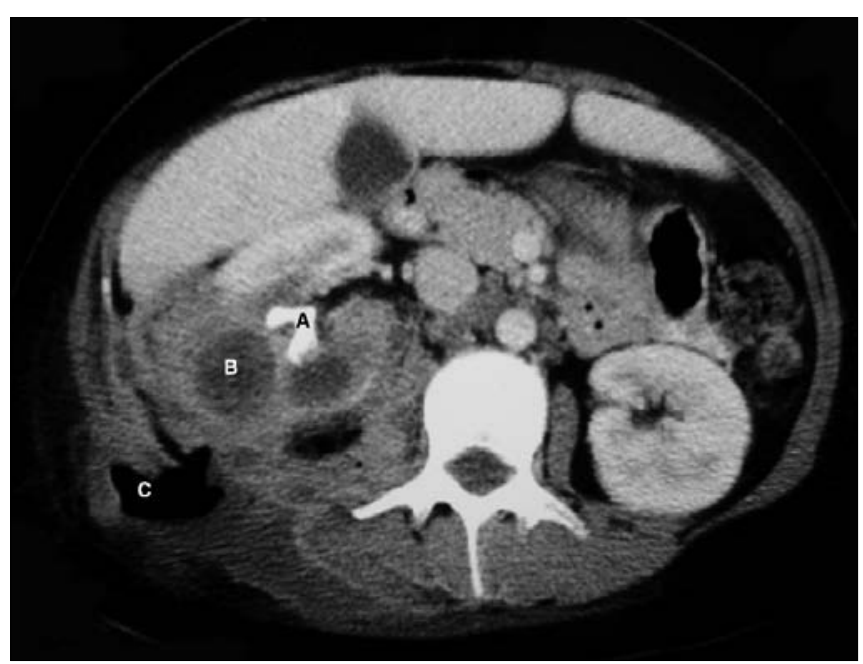

Fig. 2. Results of abdominal CT showing staghorn calculus and perinephric abscess communicating with subcutaneous abscess (post incision and drainage) 


\section{Clinical presentation}

Patients typically complain of a painful tender swelling between the ribs and the iliac crest, ${ }^{3}$ or systemic symptoms including fever, rigors, anorexia, nausea and vomiting. The presentation can be more subtle, with dysuria, hematuria, pyuria, persistent fever not responding to antibiotics, malaise, weight loss or abscesses in the chest, groin or thighs. ${ }^{3}$ Classically, patients lie supine with hips flexed to minimize irritation of the psoas muscle. Features of peritonitis or bronchial discharge of pus may occur with peritoneal or pleural perforation.

Complications of perinephric abscess include life-threatening sepsis and renal failure. Abscess extension may occur along the psoas muscle, pointing toward the groin, or more deeply into the perineum, vagina or scrotum. These abscesses may also perforate the peritoneum,${ }^{4}$ bladder, colon, spleen, ${ }^{3}$ or even erode through the diaphragm into the pleura, lungs or pericardium. ${ }^{3}$ Atelectesis, pneumonia, empyema or fistula formation with stomach, small bowel, duodenum, lung or to the skin can result.

The differential diagnosis includes renal tumours, hydronephrosis and pyelonephritis, but given the non-specific nature of symptoms, a broad differential may initially be considered.

\section{Investigation}

Pyuria is common but hematuria is present in only $6 \%-32 \%$ of patients despite the high rate of underlying urinary tract pathology. ${ }^{5}$ The white blood cell count and inflammatory markers may be elevated; however urinalysis and blood tests are neither specific nor sensitive for perinephric abscess. ${ }^{2}$

Abdominal radiographs are abnormal in up to $60 \%$ of patients, ${ }^{6}$ and suggestive features include an absent psoas border, enlarged kidney, obliteration of the renal shadow, elevation of the diaphragm with associated pleural effusion, nephrolithiasis and retroperitoneal gas. Unfortunately, these features are non-specific for renal and perirenal inflammation. Perinephric gas bubbles are highly specific, and pathognomonic of abscess.

Abdominal ultrasound is a useful and non-invasive study, but is false-negative in up to $36 \%$ of cases. ${ }^{2}$ Sonographic features of perinephric abscess include extension beyond the renal cortex with an irregular or lobulated border and longitudinal extension along the retroperitoneum. Layering of necrotic debris within the abscess cavity and irregularity of the cavity wall are helpful in differentiating abscesses from cysts. Intra-renal fluid collections can represent blood, pus or necrotic tumour, and percutaneous aspiration may be necessary to reach diagnosis.
Computerised tomography is the modality of choice it is more sensitive and more specific than ultrasound. Renal enlargement, hydronephrosis, intraparenchymal masses or Gerota's fascia thickening are non-specific findings. Perirenal fluid collection and thickening of the psoas muscle are highly suggestive, while perinephric gas is pathognomonic. The borders of the abscess may be enhanced by contrast, and analysis of the Hounsfield units of the fluid, retroperitoneal hematoma, urinoma and tumour may be differentiated from an abscess.

\section{Treatment}

Perinephric abscesses carry a significant mortality risk, particularly in the presence of sepsis, urinary tract obstruction or delayed diagnosis. Early diagnosis and drainage with antibiotic treatment is life saving. These abscesses should be managed by drainage under antibiotic coverage to reduce the risk of sepsis, although small abscesses may resolve with antibiotics alone. ${ }^{7}$ Early empirical antibiotics should be directed against the most common organisms, bearing in mind that these infections are often polymicrobial. ${ }^{3}$ Beta-lactams and aminoglycosides are appropriate initial agents, and these should be adjusted following culture. ${ }^{5}$ Percutaneous drainage and decompression of any urinary tract obstructions should be performed under ultrasound guidance; however, open surgical drainage and immediate or delayed nephrectomy may be indicated if renal function is absent or the kidney is severely infected.

\section{Comments}

Discharge to the skin, as seen in this patient, is rare, and a Medline search revealed only 3 cases of subcutaneous abscess formation secondary to a perinephric abscess. ${ }^{8-10}$ In 2 of these cases the patients were systemically unwell, while the 3rd was well, with no signs of sepsis. Similarly, our patient was systemically well with no evidence of sepsis apart from tachycardia. We speculate that the previously administered antibiotics suppressed other signs of sepsis. Subcutaneous abscesses such as that described here should bring to mind other possible intra-abdominal pathologies, such as perforated cecal carcinoma, ${ }^{11}$ hepatic abscess, ${ }^{12}$ and perforated diverticulitis. ${ }^{13}$

\section{Conclusion}

Perinephric abscesses may present with non-specific symptoms and signs. There is a strong correlation between late diagnosis and high mortality. ${ }^{14}$ Doctors need to be aware that subcutaneous abscesses in the abdomen may have underlying intra-abdominal pathology without overt signs of sepsis. 
Competing interests: None declared.

Contributors: Dylan Tsukagoshi performed the literature search and wrote the manuscript. Bozanka Dinkovski conducted a further literature search and edited the manuscript. Sunil Dasan proofread and edited the manuscript. Jalpesh Jethwa conducted a further literature search and co-wrote the manuscript.

\section{References}

1. Merimsky E, Feldman C. Perinephric abscess: report of 19 cases. Int Surg 1981;66:79-80.

2. Edelstein H, McCabe RE. Perinephric abscess. Modern diagnosis and treatment in 47 cases. Medicine (Baltimore) 1988;67: 118-31.

3. Deck AJ, Yang CC. Perinephric abscesses in the neurologically impaired. Spinal Cord 2001;39:477-81.

4. Runyon BA. Bacterial peritonitis secondary to a perinephric abscess. Case report and differentiation from spontaneous bacterial peritonitis. Am J Med 1986;80:997-8.

5. Hutchison FN. Kaysen GA. Perinephric abscess: the missed diagnosis. Med Clin North Am 1988;72:993-1014.

6. Thorley JD, Jones SR, Sanford JP. Perinephric abscess. Medicine 1974;53:441-51.

7. Dalla Palma L, Pozzi-Mucelli F, Ene V. Medical treatment of renal and perirenal abscesses: CT evaluation. Clin Radiol 1999; 54:792-7.
8. Vaidyanathan S, Hughes PL, Soni BM, et al. Unpredicted spontaneous extrusion of a renal calculus in an adult male with spina bifida and paraplegia: report of a misdiagnosis. Measures to be taken to reduce urological errors in spinal cord injury patients. BMC Urol 2001;1:3.

9. Poulos J, Johnson SR, Conrad P, et al. Dome-shaped lesion on chest radiograph: retroperitoneal abscess dissecting through the posterior chest wall. South Med J 1994;87:77-80.

10. Cowley JP, Connolly CE, Hehir M, et al. Renal carcinoma with staghorn calculus, perinephric abscess, and xanthogranulomatous pyelonephritis in same kidney. Subcutaneous abscess of thigh as initial presentation. Urology 1983;21:635-8.

11. Mann GN, Scoggins CR, Adkins B. Perforated cecal adenocarcinoma presenting as a thigh abscess. South Med J 1997;90:949-51.

12. Arslan N, Unal N, Uzuner N, et al. Pyogenic liver abscesses in a child spreading to pulmonary and subcutaneous tissues: case report. Turk J Pediatr 2004;46:279-82.

13. Peters JH, Bleichrodt RP, van Goor H. A rare manifestation of perforated diverticulitis: parastomal subcutaneous abscess. Surg Infect (Larchmt) 2003;4:227-8.

14. Salvatierra O Jr, Bucklew WB, Morrow JW. Perinephric abscess: a report of 71 cases. J Urol 1967;98:296-302.

Correspondence to: Dr. Dylan Tsukagoshi, Radiology Department, Chelsea and Westminster Healthcare NHS Trust, 369 Fulham Rd., London UK SW10 9NH; dhayashi@ doctors.org.uk 